\title{
Massive cerebral swelling immediately after cranioplasty, a fatal and unpredictable complication: report of 4 cases
}

\author{
Gill E. Sviri, MD, MSc \\ Department of Neurosurgery, Rambam (Maimonides) Medical Center, Haifa, Israel
}

Cranioplasty after decompressive craniectomy (DC) is associated with increased morbidity, but the reported mortality rate is low. Recently, some authors have reported a rare unexplained complication of sudden death in association with massive cerebral edema immediately after cranioplasty.

The author reports on 4 patients who underwent cranioplasty after DC between January 2005 and August 2010 at his department and died because of massive cerebral edema immediately after uneventful surgery and anesthesia. All 4 of the new cases reported involved young male patients who underwent decompressive hemicraniectomy after traumatic brain injury. They developed massive cerebral swelling immediately after uneventful cranioplasty (3 patients) or after removal of an epidural hematoma several hours after surgery (1 patient). All 4 patients had a large skull defect and significantly sunken craniotomy site, and all were treated with a closed vacuum suction system that was placed under the scalp and kept open at the end of the cranioplasty procedure. After surgery, the patients' pupils became fixed and dilated, and brain CT scans showed massive brain edema. Despite emergency DC, the patients did not recover, and all 4 died. A MEDLINE search showed 8 similar cases that were reported previously.

Fatal cerebral swelling after uneventful cranioplasty is a distinct clinical entity, although it is unpredictable. It is postulated that a negative pressure difference from the elimination of atmospheric pressure that had been chronically applied on the injured sinking brain in combination with the negative pressure applied by the closed subgaleal suction drain may lead to a massive brain shift toward the cranioplasty site and initiate a fatal vasomotor reaction.

http://thejns.org/doi/abs/10.3171/2014.11.JNS141152

KEY WORDS cranioplasty; traumatic brain injury; decompressive craniectomy; complication; cerebral edema; death

$\mathrm{C}$ RANIOPLASTY to restore the skull vault is performed increasingly often and is associated with a high frequency of complications compared with other brain surgery. These complications primarily include local wound infection (9\%-13\%), $, 4,4,6,7,9,10$ bone resorption (39\% in children ${ }^{7}$ ), and epidural hematoma (7.4\%). ${ }^{9}$ Nevertheless, the reported mortality rates associated with cranioplasty are low, and in most of the cases in which patients have died, death has been associated with infection and medical problems., ${ }^{2,9}$

Recently, some authors have reported a fatal complication associated with massive brain edema and death immediately after uneventful cranioplasty surgery. $2,3,5,8,11,12$ The purpose of this study was to report the history of 4 patients who developed fatal cerebral swelling after cranioplasty, review the literature for similar cases, and propose a mechanism for this rare complication of cranioplasty.

\section{Case Reports}

Between January 2005 and August 2010, four of the patients who underwent cranioplasty at Rambam (Maimonides) Medical Center in Haifa, Israel, died because of massive cerebral edema immediately after uneventful surgery and anesthesia. These patients' cases are presented below.

\section{Case 1}

A 22-year-old man was severely injured in a motor vehicle accident. His Glasgow Coma Scale (GCS) score on admission was 5, and his right pupil was dilated and unresponsive. A CT scan showed a frontal brain contusion, right subdural hematoma, and right depressed skull fracture. The patient underwent immediate DC and clot removal and was subsequently comatose for 2 months.

ABBREVIATIONS DC = decompressive craniectomy; GCS = Glasgow Coma Scale.

SUBMITTED June 16, 2014. ACCEPTED November 26, 2014.

INCLUDE WHEN CITING Published online June 19, 2015; DOI: 10.3171/2014.11.JNS141152.

DISCLOSURE The author has no personal financial or institutional interest in any of the drugs, materials, or devices described in this article. 
At 9 months after the injury, he had mild to moderate cognitive deficit, moderate left hemiparesis, and mild dependence for daily activities. Cranioplasty was performed under general inhalational anesthesia, and frozen $\left(-72^{\circ} \mathrm{C}\right)$ autologous bone was used to repair the cranial defect. The surgery (duration 95 minutes) was uneventful. A closed vacuum suction system was placed under the scalp and was applied at the end of surgery. However, the patient did not recover from anesthesia and had ineffective spontaneous breathing. Within 15 minutes after completion of cranioplasty, his pupils became dilated and unresponsive. A CT scan showed diffuse massive brain edema, especially in the contralateral hemisphere (Fig. 1). A CT angiogram showed slow flow into the ipsilateral hemisphere.

A contralateral DC was performed immediately, and the patient's brain was found to be severely swollen but not hemorrhagic. An intracranial pressure monitor was placed and showed increased intracranial pressure $(80 \mathrm{~mm} \mathrm{Hg})$. The patient did not recover, and brain death was declared after 24 hours.

\section{Case 2}

A 14-year-old male adolescent was severely injured in an all-terrain-vehicle accident. His GCS score on admission was 6 , and his left pupil was dilated and unresponsive to light. A CT scan of his brain showed a left subdural hematoma and linear occipital skull fracture. The patient underwent emergency DC and clot removal. He was comatose for 7 months, and a right ventriculoperitoneal shunt was placed at 3 months after injury because of ventriculomegaly.

At 10 months after injury, he had moderate cognitive deficit, severe right hemiparesis, and combined aphasia, and he was dependent for daily activities. Cranioplasty was performed under general intravenous anesthesia, and frozen $\left(-72^{\circ} \mathrm{C}\right)$ autologous bone was used to repair the cranial defect. The surgery (duration 100 minutes) was uneventful. A closed vacuum suction system was placed under the scalp and suction was applied at the end of surgery. However, the patient did not recover from anesthesia and had ineffective spontaneous breathing. Within 25 minutes, his pupils became dilated and unresponsive. A brain CT scan (Fig. 2) showed diffuse massive hemorrhagic cere- bral edema, especially in the contralateral hemisphere. A CT angiogram showed slow flow into the ipsilateral hemisphere.

Ipsilateral DC was performed immediately; the patient's brain was found to be severely swollen and not hemorrhagic. He did not recover, and brain death was declared after 24 hours.

\section{Case 3}

A 28-year-old man had a severe penetrating gunshot injury to the head. His GCS score on admission was 6, and both pupils were responsive to light. Brain CT scan showed a penetrating injury into the right frontal bone and lobe with intracerebral hematoma. The patient underwent emergency decompressive hemicraniectomy with removal of clots. He remained comatose for 22 days. A lumboperitoneal shunt was placed because of accumulation of CSF under the scalp, and the shunt was removed and replaced because of shunt infection. He subsequently began to demonstrate gradual motor and cognitive recovery.

At 17 months after the injury, he had mild cognitive impairment and mild left hemiparesis, and he was independent for daily activities. Cranioplasty was performed under intravenous anesthesia, and methylmethacrylate cement was used to restore the vault. The surgery and anesthesia (duration 80 minutes) were uneventful. A subgaleal drain was placed, connected to a closed vacuum suction system, and kept open at the end of the surgery.

The patient had effective spontaneous breathing and was extubated and transported to the recovery room, but he did not waken. There was no hemodynamic or hypoxic event, but both pupils become dilated and unresponsive after 20 minutes. A CT scan (Fig. 3) showed diffuse massive brain edema, especially in the contralateral hemisphere. A CT angiogram showed slow flow.

Bilateral DC was performed immediately. The patient's brain was found to be severely swollen but not hemorrhagic. He did not recover, and brain death was declared after 24 hours.

\section{Case 4}

A 24-year-old man was severely injured in an assault. His GCS score on admission was 7, and both pupils were
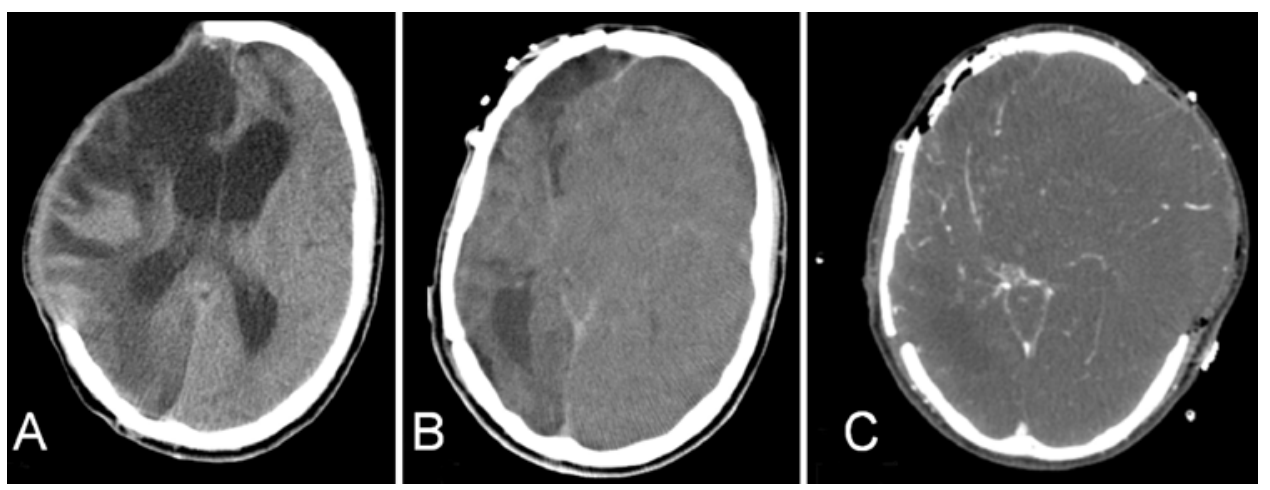

FIG. 1. CT axial images obtained in a 22-year-old male patient treated with DC after traumatic brain injury. A: Image obtained 3 months after injury and 5 months before cranioplasty. (No image from the preoperative CT study was available.) At the time of the cranioplasty procedure, the scalp was significantly sunken. B: Image obtained immediately after cranioplasty showing massive edema and brain shift toward the side of the cranioplasty. C: Image obtained after salvage decompressive surgery showing massive cerebral edema. 

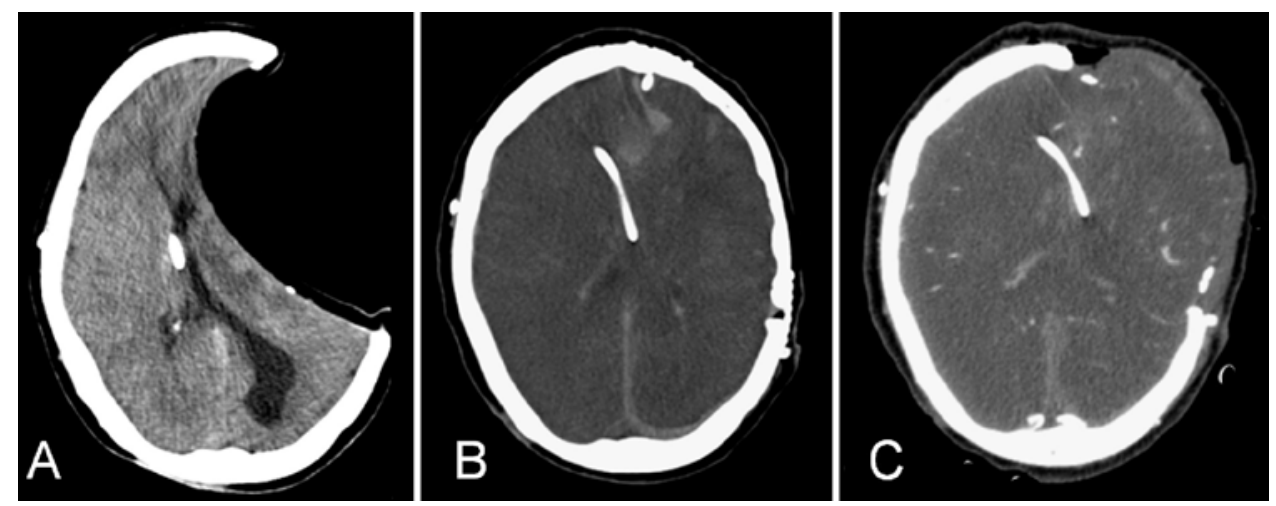

FIG. 2. CT axial images obtained in a 14 -year-old male patient treated with DC after traumatic brain injury. A: Image obtained immediately before cranioplasty. B: Image obtained immediately after cranioplasty showing massive hemorrhagic edema and brain shift toward the side of the cranioplasty. C: Image obtained after salvage decompressive surgery showing massive cerebral edema.

responsive to light. A CT scan showed a skull fracture, left subdural hematoma, and midline shift. The patient underwent emergency decompressive hemicraniectomy and removal of clots. He was comatose for 2 weeks but subsequently demonstrated motor and cognitive recovery.

At 3 months after the injury, he had cognitive deficit, motor dysphasia, and moderate right hemiparesis, and he was dependent for daily activities. Cranioplasty was performed under general inhalational anesthesia, and frozen $\left(-72^{\circ} \mathrm{C}\right)$ autologous bone was used to repair the cranial defect. The surgery (duration 70 minutes) was uneventful. A subgaleal drain was placed, connected to a closed vacuum suction system, and kept open at the end of the surgery.

The patient recovered from surgery and was awake and oriented. At 4 hours after surgery, he vomited and complained of headache. A CT scan (Fig. 4) showed a large epidural hematoma under the reconstructed vault, with compression of the left ipsilateral hemisphere. He had immediate surgery to remove epidural clots, and the autologous bone flap was replaced. The surgery and anesthesia were uneventful, and he had normal measured hemodynamic and metabolic parameters. A subgaleal drain was placed, connected to a closed vacuum system, and kept
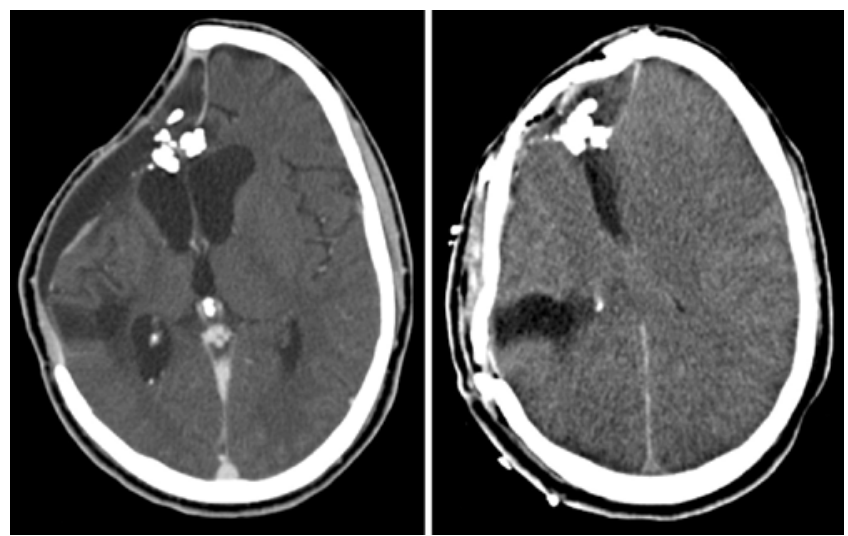

FIG. 3. CT axial images obtained in a 28 -year-old male patient treated with DC after penetrating traumatic brain injury. Left: Image obtained immediately before cranioplasty. Right: Image obtained immediately after cranioplasty showing massive edema and brain shift toward the side of the cranioplasty. open at the end of surgery. The patient, however, did not recover from anesthesia. Within 90 minutes, his contralateral pupil became dilated and unresponsive. A CT scan (Fig. 4) showed diffuse massive brain edema, especially in the contralateral hemisphere. A CT angiogram showed slow flow into the ipsilateral hemisphere.

Bilateral DC was performed immediately, and an external ventricular drain was placed; the patient's brain was found to be severely swollen but not hemorrhagic. Brain death was declared within 24 hours. An autopsy showed necrotic brain tissue without any signs of major arterial or venous occlusion.

\section{Literature Search}

A MEDLINE search was performed to identify articles of interest published from 1988 to 2013. The following key words were used: "cranioplasty," "cranial defect," "craniectomy," "bone flap," "complication," "death," and "mortality." The search was limited to articles in the English language. The reference sections of the identified studies were reviewed to identify any additional relevant articles. The search revealed 8 cases of unexplained cerebral swelling and death immediately after uneventful cranioplasty in patients who had undergone DC. In 5 cases the patients had suffered traumatic brain injury, and in the other 3 cases they had suffered middle cerebral artery infarction. Data on clinical characteristics and outcome of the cases were extracted from the reports and tabulated (Table 1).

\section{Discussion}

All 4 of the cases reported in the present paper involved young male patients who had undergone decompressive hemicraniectomy after traumatic brain injury and had subsequently undergone uneventful surgery and anesthesia for restoration of the skull vault. All of the patients had a significantly sunken craniotomy site with atrophy and encephalomalacia at the side of the DC. None of the patients recovered from the cranioplasty surgery, and all 4 died. The patient in Case 4 was exceptional because he developed massive brain edema after removal of an epidural hematoma that had complicated the cranioplasty surgery.

Eom et al. ${ }^{3}$ reported on a 63 -year-old man who under- 

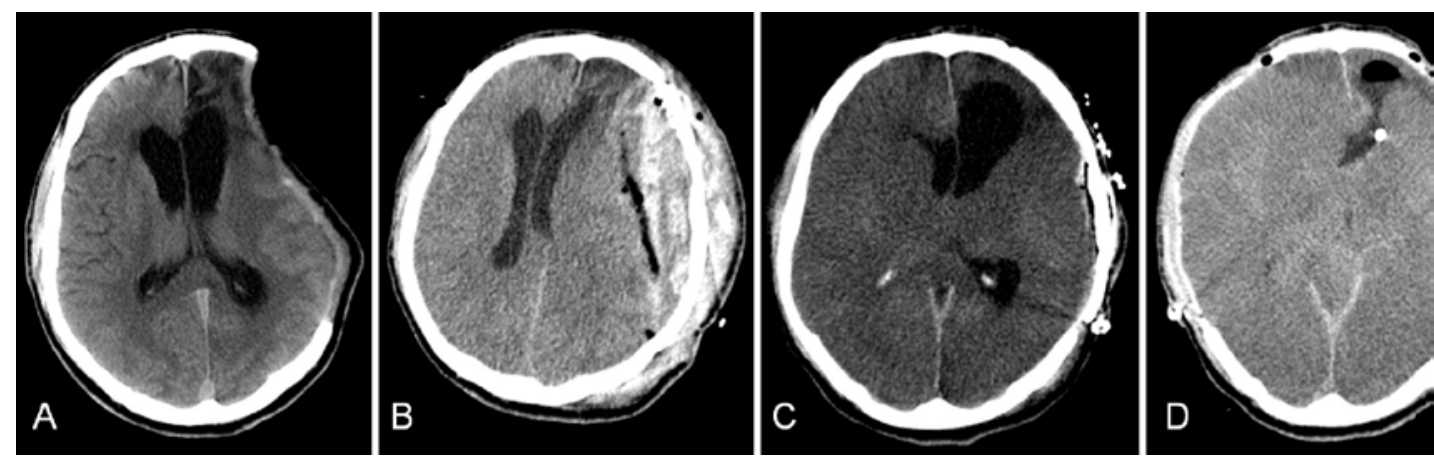

FIG. 4. CT axial images obtained in a 22-year-old male patient treated with DC after traumatic brain injury. A: Image obtained immediately before cranioplasty. B: Image obtained several hours after cranioplasty showing a left epidural hematoma and hematoma under the left scalp as well as brain shift. C: Image obtained immediately after removal of epidural clots showing massive edema and brain shift toward the side of the cranioplasty. D: Image obtained after salvage bilateral decompressive surgery and placement of an external left frontal ventricular drain. Massive bilateral cerebral edema is evident.

went decompressive hemicraniectomy after a right middle cerebral artery stroke; several months after the stroke, he had an autologous bone flap replacement with an uneventful surgical and anesthetic course. ${ }^{3}$ Nevertheless, he did not recover from anesthesia, and a brain CT scan performed 3 hours after surgery showed severe brain swelling with hemorrhage that affected the basal area. The mechanism of this complication was unknown because cerebral angiography showed no arterial or venous occlusion, but the authors attributed the bleeding to possible deep vein occlusion or congestion that caused bilateral hemorrhagic infarctions, impaired autoregulation in the chronically infarcted brain, and increased cerebral blood flow caused by the cranioplasty. ${ }^{3}$

A similar hemodynamic mechanism was suggested by Honeybul et al., ${ }^{5}$ who reported on 3 young male patients who had massive cerebral edema immediately after uneventful cranioplasty. These patients had undergone bifrontal DC for treatment of traumatic brain injury, and they subsequently underwent uneventful autologous cranioplasty. However, the patients did not wake up from the surgery; within 1 to 2 hours, they developed tachycardia and hypertension, both pupils became fixed and dilated. CT scans showed massive cerebral edema.

Santana-Cabrera et al. ${ }^{8}$ reported on a 17 -year-old male who had undergone bifrontal DC for traumatic brain injury. Two months later, he underwent cranioplasty with uneventful surgery and anesthesia, but he did not wake up from anesthesia. He developed hypertension and bradycardia, and both pupils became fixed and dilated. A CT scan showed massive cerebral edema. Although reoperation was performed immediately afterward, with an expanded craniotomy and bifrontal lobectomy, the patient died 12 hours later.

Chitale et al. ${ }^{2}$ reported on a 64-year-old man who underwent cranioplasty with polyetheretherketone, or PEEK, allograft 1 year after undergoing DC because of massive right middle cerebral artery stroke. After awakening from an uneventful operation, he had a generalized tonic-clonic seizure. A CT scan showed postoperative changes and reexpansion of the right cerebral hemisphere, but a followup CT scan 5 hours later showed diffuse brain edema, and the patient subsequently died.
Zebian and Critchley ${ }^{12}$ reported on a 40-year-old woman who underwent a left-side DC because of a malignant middle cerebral artery infarct. She recovered partially, and 2 years later she underwent an uncomplicated titanium cranioplasty and placement of a subgaleal drain on full vacuum. Immediate after surgery, she had 2 seizures, and her pupils became fixed and dilated. A CT scan showed a massive shift of the brain toward the cranioplasty and brainstem hemorrhage. A decision was made not to intervene, and the patient died.

A possible mechanism for massive cerebral edema was suggested by Van Roost et al. ${ }^{11}$ The authors reported on 17 cases of unexplained diffuse postoperative brain edema after 12,000 uneventful neurosurgical procedures in several centers in Germany and Switzerland. There was 1 patient who had cranioplasty with a similar course as those observed in the present study. This young male patient had previously undergone a decompressive hemicraniectomy for treatment of traumatic brain injury; the cranioplasty surgery and anesthesia were uneventful, but he did not recover from anesthesia and died. A CT scan showed bilateral brain edema. After eliminating different possible causes of brain swelling, such as hemodynamic, ischemic, and metabolic causes, the authors attributed the swelling to intracranial hypotension caused by the loss of CSF from the wound and a subgaleal vacuum drain. After cranioplasty, the patient had lost $390 \mathrm{ml}$ of fluid from the suction drain before the pupils became fixed and dilated. ${ }^{11}$

In the 4 cases presented in the current report, it is possible that a negative pressure difference may have caused cerebral edema. The application of a vacuum suction system to the large cavity at the reconstructed cranial vault at the end of the cranioplasty may have caused intracranial hypotension and massive shifting of the chronically depressed brain. All 4 patients had a large bone defect with depressed scalp and marked brain atrophy at the side of the craniotomy, which may have increased susceptibility to developing intracranial hypotension. The elimination of atmospheric pressure with the suction drain may have caused a negative pressure difference and shifting of the depressed brain to the reconstructed cranial vault, and this pressure difference also may have caused secondary ischemia and brain swelling. 


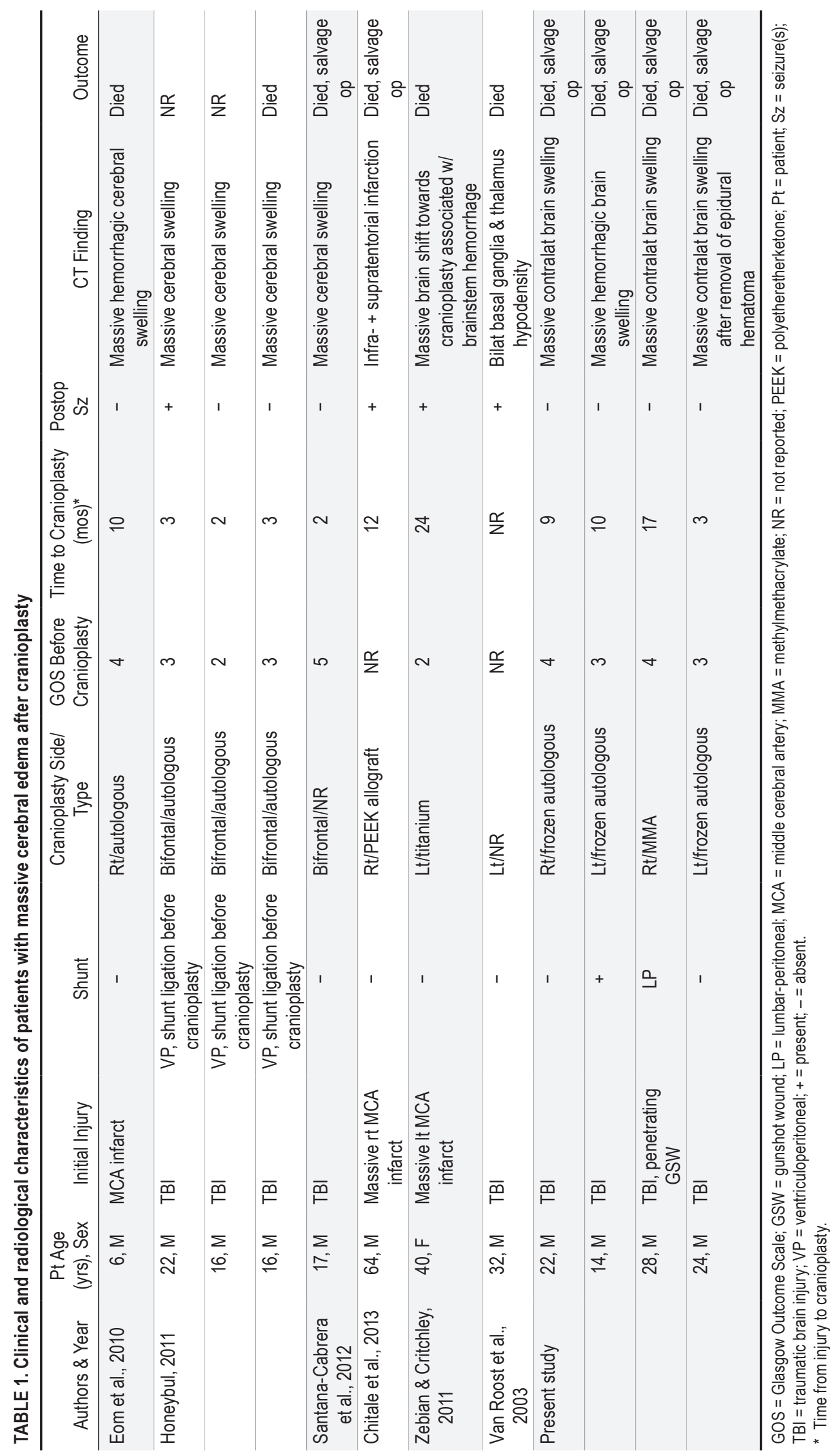


In Case 4, the initial surgery for cranioplasty was uneventful, and the patient awoke after anesthesia. He developed an epidural hematoma in the space between the reconstructed vault and the depressed brain, and he had subsequent shift of the brain. The epidural clots further depressed the ipsilateral hemisphere. The patient was awake when he was intubated for the second operation, so it is likely that the epidural hematoma did not have a major effect on the development of postoperative swelling. However, the epidural clots increased the space between the brain and the reconstructed vault. Clot removal and concomitant elimination of atmospheric pressure because of the vacuum drain may have caused marked brain shifting, ischemia, and secondary swelling.

Nevertheless, this postulated negative pressure mechanism provoking a fatal vasomotor reaction is speculative. Initially after the first case (Case 1) in May 2005, it was believed that the massive brain swelling was caused by venous occlusion and that the ipsilateral transverse sinus might have been occluded from the initial injury and the contralateral jugular vein could have been occluded during positioning. There was no imaging to confirm this hypothesis, but care was subsequently taken in positioning patients to ensure that the contralateral jugular vein was not compressed. In Case 3, preoperative CT venography showed an occluded ipsilateral transverse sinus, and pins were used in positioning to ensure a more natural position of the neck.

Van Roost et al. ${ }^{11}$ estimated that a negative pressure difference may occur in 1 of 7000 neurosurgical operations for supratentorial lesions. The incidence of this pressure difference after cranioplasty may be higher, especially when the cranial vault defect is large and the brain is atrophic and depressed. Based on the literature review and the present cases, fatal massive cerebral edema immediately after uneventful cranioplasty may be a distinct clinical syndrome. The 4 cranioplasty fatalities reported in the present paper occurred between May 2005 and August 2010, when 57 cranioplasty procedures were performed at our institution using the same operative technique, including the use of a closed vacuum suction drainage system (12- and 14-Fr Hemovac, Zimmer) that was kept in place for 24 to 48 hours after the operation. Since the most recent case, in August 2010, we do not apply suction to the drain at the end of surgery, but only in the recovery room after the patient has fully awakened, and we have had no further recurrence of this complication in 76 cranioplasty operations performed between September 2010 and $\mathrm{Au}-$ gust 2014.

\section{Conclusions}

Fatal massive cerebral edema is an uncommon complication after cranioplasty in patients who had previously undergone DC. This complication is unexplained but may be attributed to concomitant elimination of atmospheric pressure on the depressed brain and a negative pressure change caused by suction applied via a subgaleal vacuum drain at the end of cranioplasty. The postulated negative pressure change may cause brain shifting and may markedly decrease cerebral blood flow. This complication occurred immediately after cranioplasty and was fatal and unpredictable. Therefore, it is recommended that neurosurgeons should be aware of this clinical syndrome, discuss it with the patient and family, and avoid application of suction via the subgaleal drain after cranioplasty until the patient is fully awake.

\section{References}

1. Chang V, Hartzfeld P, Langlois M, Mahmood A, Seyfried D: Outcomes of cranial repair after craniectomy. J Neurosurg 112:1120-1124, 2010

2. Chitale R, Tjoumakaris S, Gonzalez F, Dumont AS, Rosenwasser RH, Jabbour P: Infratentorial and supratentorial strokes after a cranioplasty. Neurologist 19:17-21, 2013

3. Eom KS, Kim DW, Kang SD: Bilateral diffuse intracerebral hemorrhagic infarction after cranioplasty with autologous bone graft. Clin Neurol Neurosurg 112:336-340, 2010

4. Gooch MR, Gin GE, Kenning TJ, German JW: Complications of cranioplasty following decompressive craniectomy: analysis of 62 cases. Neurosurg Focus 26(6):E9, 2009

5. Honeybul S: Sudden death following cranioplasty: a complication of decompressive craniectomy for head injury. Br J Neurosurg 25:343-345, 2011

6. Mukherjee S, Thakur B, Haq I, Hettige S, Martin AJ: Complications of titanium cranioplasty - a retrospective analysis of 174 patients. Acta Neurochir (Wien) 156:989-998, 2014

7. Rocque BG, Amancherla K, Lew SM, Lam S: Outcomes of cranioplasty following decompressive craniectomy in the pediatric population. J Neurosurg Pediatr 12:120-125, 2013

8. Santana-Cabrera L, Pérez-Ortiz C, Rodríguez-Escot C, Sánchez-Palacios M: Massive postoperative cerebral swelling following cranioplasty. Int J Crit Illn Inj Sci 2:107-108, 2012

9. Stephens FL, Mossop CM, Bell RS, Tigno T Jr, Rosner MK, Kumar A, et al: Cranioplasty complications following wartime decompressive craniectomy. Neurosurg Focus 28(5):E3, 2010

10. Thavarajah D, De Lacy P, Hussien A, Sugar A: The minimum time for cranioplasty insertion from craniectomy is six months to reduce risk of infection-a case series of 82 patients. Br J Neurosurg 26:78-80, 2012

11. Van Roost D, Thees C, Brenke C, Oppel F, Winkler PA, Schramm J: Pseudohypoxic brain swelling: a newly defined complication after uneventful brain surgery, probably related to suction drainage. Neurosurgery 53:1315-1327, 2003

12. Zebian B, Critchley G: Sudden death following cranioplasty: a complication of decompressive craniectomy for head injury. Br J Neurosurg 25:785-786, 2011

\section{Correspondence}

Gill E. Sviri, Department of Neurosurgery, Rambam (Maimonides) Health Care Campus, HaAliya HaShniya St. 8, Haifa, Israel.email: g_sviri@rambam.health.gov.il. 